\title{
Energy Analysis in Wireless Sensor Network: A Comparison
}

\author{
Vasaki Ponnusamy \\ Faculty of Integrative Sciences and Technology, Quest International University Perak, Jalan Raja \\ Permaisuri Bainun, 30250 Ipoh, Malaysia \\ E-mail: vasaki.ponnusamy@gmail.com
}

\begin{abstract}
This paper explores energy exploitation in a sensor network deployed under diverse configurations. We are looking at sensor network deployed using multi hop, clustering, direct and mobility based transmission. A lot of work has been done using these mechanisms to convey sensor data to the base station by taking energy into consideration. The aim of this study is to present an analysis of energy utilization using these techniques by comparing energy usage at different parts of the network. The outcome of the analysis helps researchers to identify the best mechanism for energy efficient communication in sensor network. Further, the research presents a comparison of work done by other researchers using these communication techniques, to further validate our analysis.
\end{abstract}

Keywords: Energy Analysis, Comparison of Energy Utilization, Wireless Sensor.

\section{INTRODUCTION}

Sensor networks consist of limited batterypowered nodes deployed to sense the target. Replacing or recharging these nodes is almost impossible as the deployed target areas are often difficult to access. The design of sensor nodes has to be compromised in terms of size, cost and battery size. Larger batteries can last longer but yields higher cost and bigger nodes, whereas smaller batteries have shorter lifespan and lesser cost [1]. Researchers are looking into various options of extending the lifespan of sensor batteries from the design, manufacturing, protocol and architecture aspects [2]. In this work, we are limiting our research to the protocol and architecture aspects only. According to many researchers, uneven energy consumption becomes the major factor for routing hole in the network [3, $4,5]$. Many researches have been conducted for efficient handling of energy in sensor networks from the architectures and protocols point of view.

Energy aware routing, shortest path routing, clustering, data aggregation, mobility based routing and direct transmission are among some of the possibilities researchers are looking into in order to reduce energy consumption.

Our main focus in this research is to mathematically analyse energy consumption of these architectures and perform a comparison of simulation results conducted by other researchers, and finally evaluate further with our simulation results. The analysis is limited to energy aspect only by comparing energy utilization rate at different regions of the network. The goal is to look into the best deployment option from the energy utilization perspective. Further, the research provides a critical reasoning for adopting other alternative mechanisms than relying solely on traditional routing.

The organization of the rest of this paper is as follows. General analytical model is discussed in section 2 followed by energy analysis using various mechanisms. In Section 3, we compare the simulation results using these different mechanisms. The paper is then concluded in section 4. 


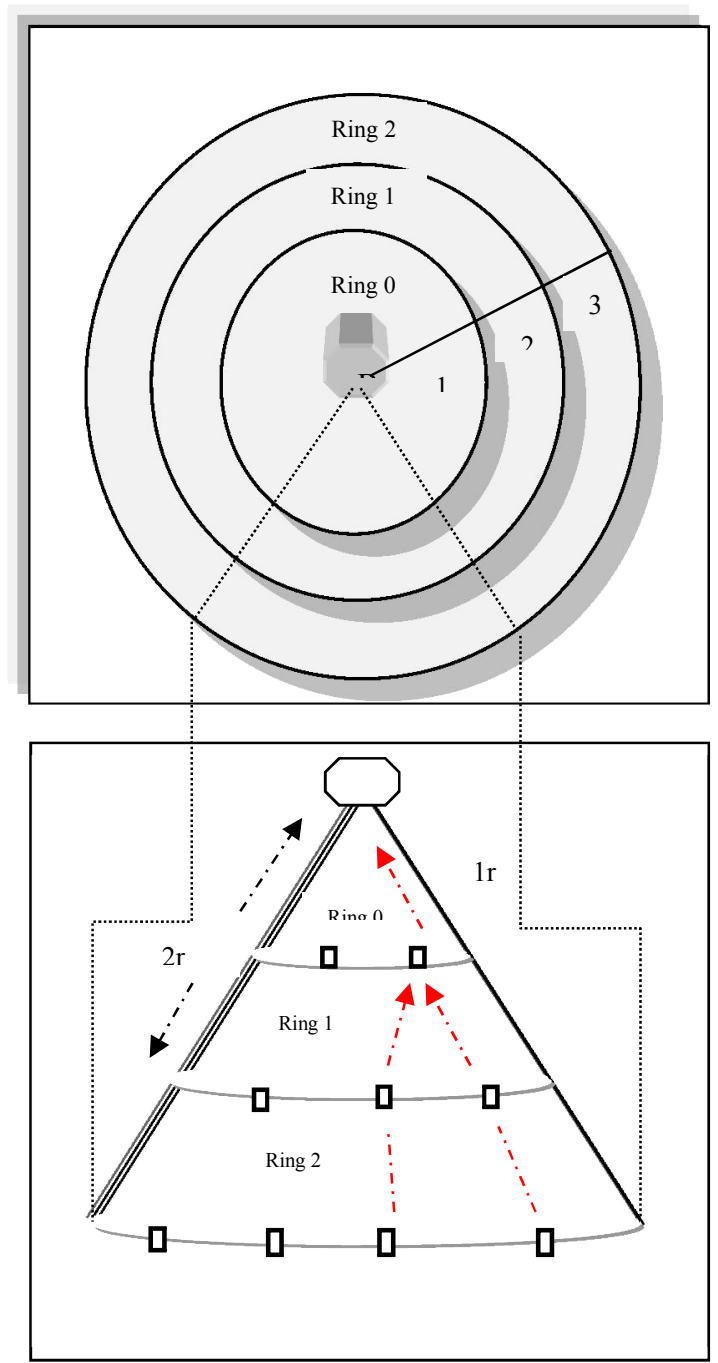

Fig. 1 Uniform distribution of sensor network in a ring architecture

\section{ANALYTICAL MODEL}

In our work we assume the homogeneous sensor network is deployed in a random fashion with uniform distribution of sensor nodes. The deployment area consists of a circle with radius $r$ and the ring is located in a rectangle with $\mathrm{L} \times \mathrm{L}$ square area. The base station is assumed to be located in the centre of the circle and the circle is partitioned into $\mathrm{m}$ rings, namely ring 0 , ring 1 till ring $\mathrm{m}$. The distance between two adjacent rings is $\mathrm{r}$ meter and the total area consists of $\mathrm{L}=\mathrm{m} \times \mathrm{r}$ meters. Ring 0 has radius of $r$ meters, ring 1 with $2 r$ meters and ring 2 with $3 \mathrm{r}$ meters respectively. It is assumed that the sensor nodes are randomly placed on the rings. As can be seen in Figure 1, a piece of quadruple is partitioned and displayed in the next figure to show how sensors are placed on the ring and how sensor data is routed depending on the mechanism, multihop, clustering, direct transmission or using mobile agent.

In Wireless Sensor Network, energy is consumed at many parts of the networks. They are mainly energy consumed for message sending, message receiving and data processing or sensing of event. This energy model is extracted from $[6,7]$ and LEACH [8] also considers similar model for its first order radio model. The simplified energy consumption model is defined as given below:

Energytrans $=$ Energyelec $X a+\quad$ Energyamp $X d X a$

Energyrecv $=$ Energyelec $X a$

Energysense $=$ Energycpu $X$ a

Whereby a is the data rate or length of data packet, $\mathrm{d}$ is the transmission distance, and some typical values for the parameters are [8]:

Energy $_{\text {cpu }}=60 \times 10^{-9} \mathrm{~J} / \mathrm{bit}$,

Energy $y_{\text {elec }}=45 \times 10^{-9} \mathrm{~J} / \mathrm{bit}$,

Energy $y_{\text {amp }}=10 \times 10^{-12} \mathrm{~J} / \mathrm{bit} / \mathrm{m}^{2}($ when $n=2)$,

or, Energy $y_{\text {amp }}=0.001 \times 10^{-12} \mathrm{~J} / \mathrm{bit} / \mathrm{m}^{4}($ when $n=4)$,

Energy $_{\text {elec }}=135 \times 10^{-9} \mathrm{~J} / \mathrm{bit}$

Assuming that,

Energyelec $=\mathbb{E}$, Energyamp $=A$,

Energycpu $=\mathbb{C}$

Total Energy Consumption $=$ Energytrans + Energyrecv + Energysense

$=$ Energyelec $X a+$ Energyamp $X d 2 X a+$ Energyelec $X a+$ Energycpu $X a$

Therefore,

Total Energy Consumption

$=a\left(2 \mathbb{E} c+A X d^{2}+\mathbb{C}\right)$

As can be seen in Eq (1), total energy utilization in sensor network is directly proportional to the length of data packet. Energy consumption increases with the increase in data packet size and vice versa. Thus if the length of data packet is reduced, total energy consumption is reduced as well. This shows that packet size plays one of the major functions in energy utilization of sensor network. A lot of work has been done to reduce data packet size by performing compression [9], data aggregation [10, 11], data packet size optimization [12] and etc. Therefore an optimum packet size is crucial in order to preserve energy at sensor nodes.

Besides that, total energy consumption is also proportional to $\mathrm{d} 2$ and $\mathrm{d} 4$, where $\mathrm{d}$ is the transmission distance [6,7]. Therefore, transmission distance should also be looked into in order to 
reduce energy consumption in sensor network. A lot of effort has been made to reduce transmission distance by exploiting cluster head [8, 11, 13], mobile entity/relay $[14,15,16,17,18]$ and mobile base station [5, 19]. These approaches reduce transmission distance between the transmitter and receiver so that the transmitter amplifier energy can be reduced.

In comparison to the previous work, our analysis aims at 5 different approaches for energy consumption using i. multihop routing, ii. clustering, iii. direct transmission, iv. mobile agent with cluster head, and v. mobile agent with direct transmission (Figure 2). We are analyzing the energy usage at different rings in the network as ring 0 , as shown in Figure 1, is assumed to be consuming more energy than other parts of the ring since it has to forward data coming from other parts of the network besides transmitting its own data (for traditional routing). Since the base station is placed at the centre of the ring, the network around this region is often more congested than other parts of the network. Therefore we are analyzing energy consumption at two different parts of the network namely ring 0 and ring 1 , to enable a comparison and show how multihop routing burdens the nodes closer to the base station and yields to shorter lifetime. Also this analysis helps to justify how other alternative approaches such as clustering and mobility can yield a better lifetime compared to traditional routing.

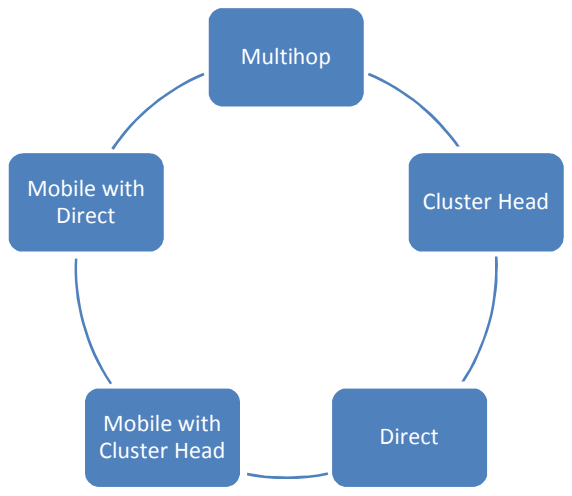

Fig. 2. Comparison of different energy usage mechanisms

\subsection{Multihop Communication}

Traditional multihop routing (Figure 3) works by having intermediate nodes forwarding data coming from other parts of the network [3, 4, 20, 21, 22]. Nodes away from the base station do not directly forward sensor data to the base station. As seen in Figure 1, nodes in ring 2 relay data to the intermediate nodes in ring 1 , followed by ring 0 and finally to the base station. Therefore nodes around the base station have to relay more data and cause significant load around ring 0 . This also causes uneven energy consumption at different parts of the network and this becomes the main factor for energy hole. An analytical model was 
presented by [9], that looked into total energy consumption per node at different parts of the network.

According to the author, nodes in ring 0 have to relay more traffic and have shown the per node traffic at ring 0 , ring 1 and ring i. In our analysis, we consider similar model as [9] and have further devised a mathematical analysis for other aspects of using mobile agent and clustering.

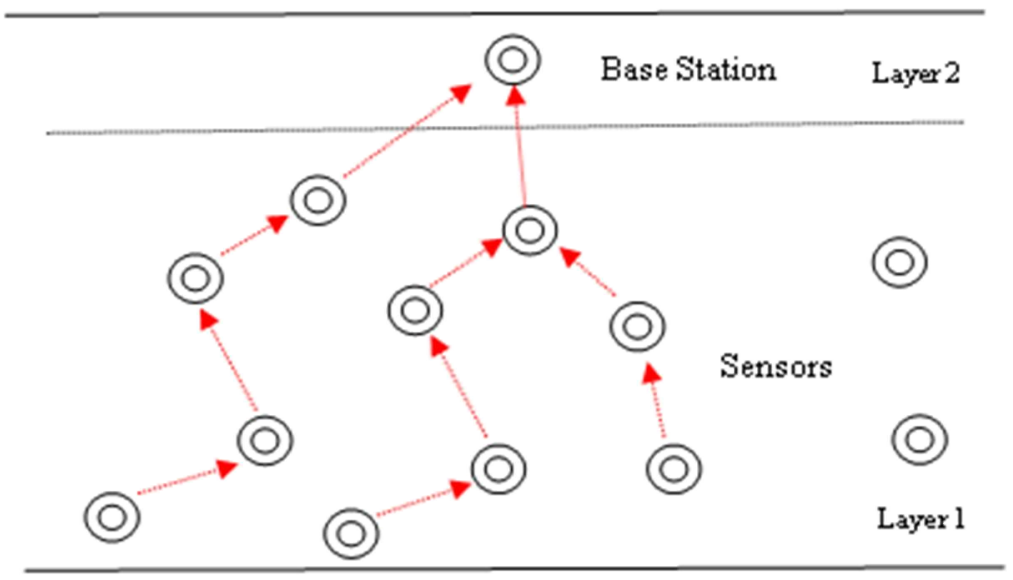

Fig. 3 Multihop communication

Energy consumption in ring 0 is

$$
\begin{aligned}
& =\left(L^{2}-1\right) E X a+\left(L^{2}\right) E+\# p X d^{2} X a+\mathbb{C} X a \\
& \pi r^{2} \quad \pi r^{2}
\end{aligned}
$$$$
=a\left(L^{2}\right) E c-E-\left(L^{2}\right) E c+\left(L^{2}\right) A X d^{2}+\mathbb{C}
$$

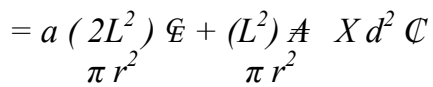

Energy consumption in ring 1 is

$$
=a\left(2 L^{2}-5 \pi r^{2}\right) E+L^{2}-\pi r^{2} A X d^{2}+\mathbb{C}
$$

As can be seen in Figure 4, total energy consumed per node at ring 0 is much greater than ring 1 due to the factors discussed above. As the network width increases, energy consumption at ring 0 increases as observed in Eq (1), since total energy consumption is dependent on ( $2 \mathrm{~L} 2$ ) as compared to ( $2 \mathrm{~L} 2-5 \pi \mathrm{r} 2$ ) in Eq (2) at ring 1 of the network. Increase in network width causes increase in transmission distance between nodes.

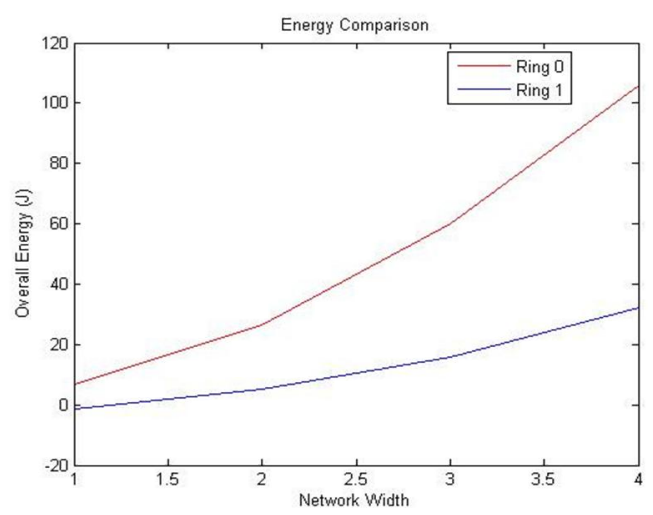

Fig. 4 Total energy consumption at ring 0 and 1 using multihop architecture

\subsection{Clustering Communication}

Clustering is one of the energy conserving mechanisms proposed in sensor network [8]. In this architecture, sensor nodes are grouped into clusters using respective clustering mechanism such as LEACH [8], [11] and [13]. As seen in Figure 5, a cluster head is elected in each cluster to collect or aggregate data from other nodes within the same cluster, while sensor nodes are only responsible for sensing the event and relaying data to the cluster 
A. Magdy et. al / International Journal of Computer Networks and Communications Security, 2 (9), September 2014

head. Thus, remarkable energy is saved as sensor nodes do not take part in routing and this is proven by LEACH. Cluster head consumes energy for sensing or processing, transmission of data to the base station and receiving data from sensor nodes. Eq ( $3 \& 4$ ) present the energy consumption by a cluster head.

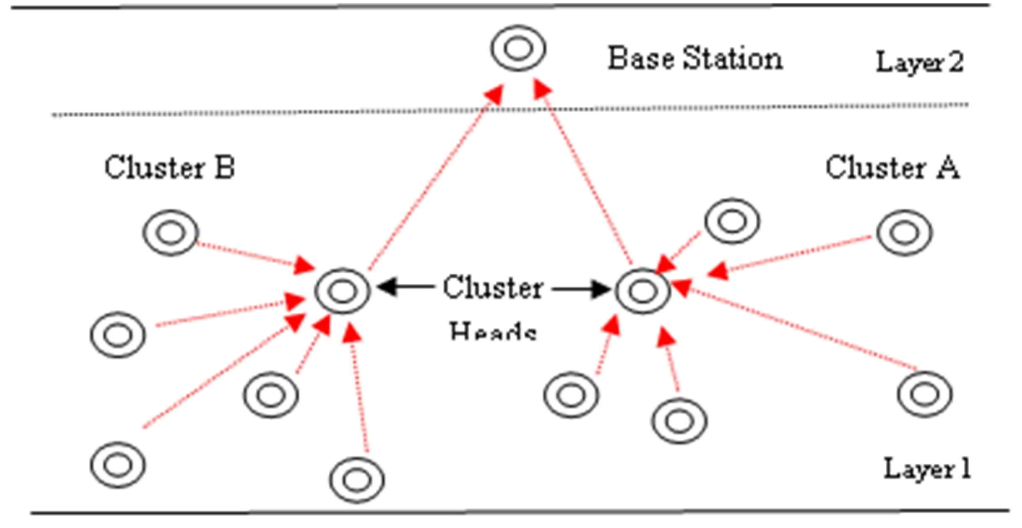

Fig. 5 Clustering communications

Energy consumption in ring 0 is

$=a\left(2 \pi L r^{2}\right) E c+\left(\pi L r^{2}\right) A X d^{2}+\mathbb{C}$

$=a\left(2 \pi L r^{2}\right) E+\left(\pi L r^{2}\right) A X d^{2}+\mathbb{C}$

$\approx a\left(A X d^{2}+2 \mathscr{E}+\mathbb{C}\right)$

Energy consumption in ring 1 is

$=a\left(6 \pi r^{2}\right) E+\left(3 \pi r^{2}\right) \nexists X d^{2}+\mathbb{C}$

$=a\left(6 \pi L r^{2}\right) E+\left(3 \pi r^{2}\right) \nexists X d^{2}+\mathbb{C}$

\subsection{Direct Communication}

Direct transmission is employed by transmitting data directly to the base station (Figure 6). Intermediate nodes are eliminated in this routing mechanism. Direct transmission involves energy consumption for sensing and transmission only.

Energy is not consumed for receiving as direct transmission does not involve receiving data from any other nodes. As presented in Eq (5) and Eq (6) for energy consumption at ring 0 and ring 1 respectively, direct transmission consumes less energy as compared to multihop. But this is only practical for nodes closer to the base station as signal decays as the 2nd power of the distance $[6,7]$. Moreover higher energy will be consumed for nodes further away from the base station as total energy consumption is directly proportional to the transmission distance as discussed above. As can be seen in Eq (5) and Eq (6), energy consumption in ring 1 is four times more than in ring 0 and it increases linearly as the transmission distance $d$ increases. Energy consumption will be proportional to $\mathrm{d} 4$, after the threshold value of transmission distance $[6,7]$. 
A. Magdy et. al / International Journal of Computer Networks and Communications Security, 2 (9), September 2014

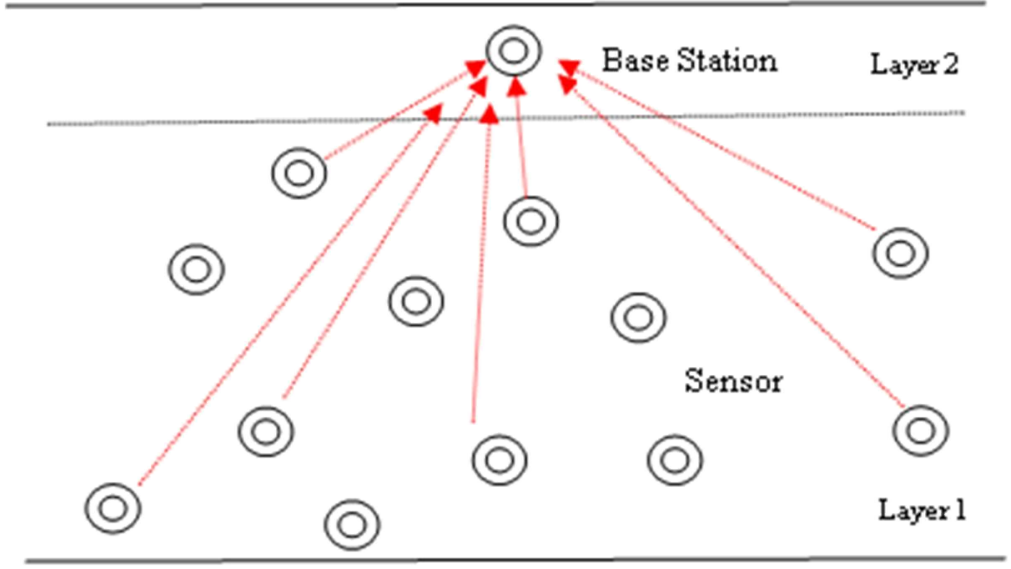

Fig. 6 Direct communication

Energy consumption in ring 0 is

Total Energy Consumption = Energytrans + Energysense

$=A X d^{2} X a+\mathscr{E} X a+\mathbb{C} X a$

$=a\left(\nexists X d^{2}+E+\mathbb{C}\right)$

Energy consumption in ring 1 is

Total Energy Consumption = Energytrans + Energysense

$=a\left(A X(2 d)^{2}+\mathscr{E}+\mathbb{C}\right)$

$=a\left(A X 4 d^{2}+\mathbb{E}+\mathbb{C}\right)$

\subsection{Mobile Communication}

Mobility in sensor network can be employed by having a mobile base station, a mobile entity forwarding data to the base station (Figure 7), or a mobile entity collecting data from the cluster head (Figure 8). In our analysis, a mobile entity will be moving near the proximity of sensor nodes, and the transmission of data is assumed to occur within a shorter distance, $\mathrm{H}$ in this case. The transmission distance, $\mathrm{H}$ is smaller than the transmission distance, $\mathrm{d}$ in other mechanisms discussed above.
This is possible and has been proven in [2] in which an airplane flying meters above the sensor field was able to retrieve data from the static sensor nodes. Total energy consumption using mobile entity is feasible by using two approaches either by using a cluster head $[23,24]$ or by a sensor directly forwarding to a mobile entity or agent [2]. Our analysis, as shown in Eq (7) and Eq (8), proves that mobility based approach reduces the transmission distance between the sender and receiver. As discussed in section 2, transmission distance is proportionally related to total energy consumption. Using a cluster head, sensor nodes forward data to the cluster head and the cluster head is responsible for forwarding data to the mobile agent. Whereas using a direct approach, mobile agents receive data directly from the sensor nodes. Based on the mathematical analysis and simulation results, employing clustering mechanism for forwarding data to the mobile entity produces better results compared to direct approach. This is because in clustering mechanism only the cluster head will be communicating with the mobile entity, while all the member nodes within the same cluster are dedicated only for sensing. Tremendous amount of energy is saved within these nodes rather than all the nodes communicating with the mobile entity. $[23,24]$ looked into such mechanism of employing clustering and mobile entity for energy efficient communication. 
A. Magdy et. al / International Journal of Computer Networks and Communications Security, 2 (9), September 2014

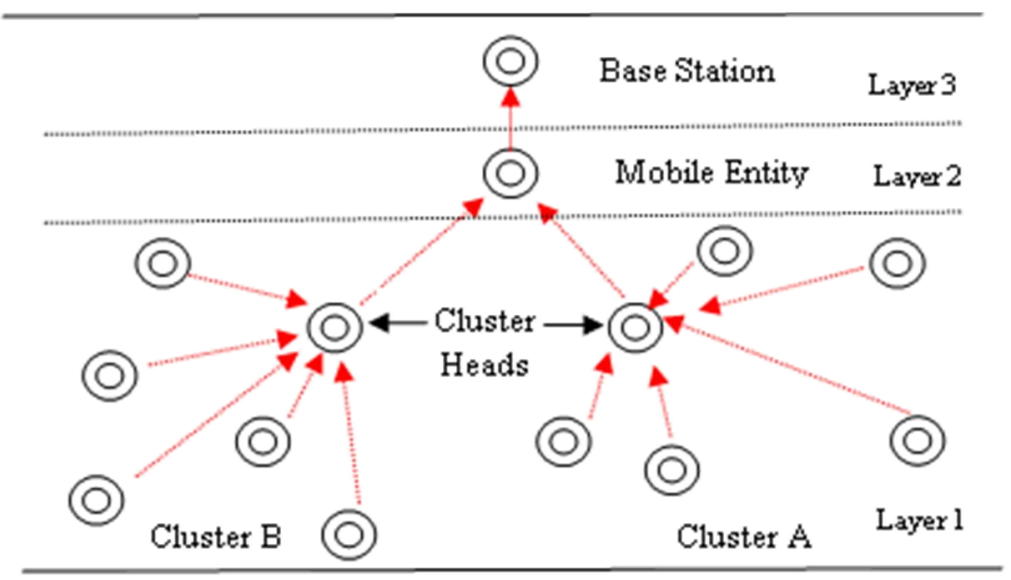

Fig. 7 Mobility with clustering communication

Energy consumption in ring 0 is

$=\frac{\left(2 \pi L r^{2}\right)}{\mathrm{N}^{2}} E X a+\frac{\left(\pi L r^{2}\right)}{\mathrm{N}^{2}} \# X H^{2} X a$

$\left.=a \frac{\left(2 \pi L r^{2}\right)}{\mathrm{N}^{2}} E+\frac{\left(\pi L r^{2}\right)}{\mathrm{N}^{2}} \neq X H^{2}\right)$

where $\mathrm{H}<\mathrm{r}$

Energy consumption in ring 1 is

$$
\left.=a \frac{\left(6 \pi L r^{2}\right)}{\mathrm{N}^{2}} E+\frac{\left(3 \pi r^{2}\right)}{\mathrm{N}^{2}} A X H^{2}\right)
$$

where $\mathrm{H}<\mathrm{r}$

\subsection{Mobile with Direct Communication}

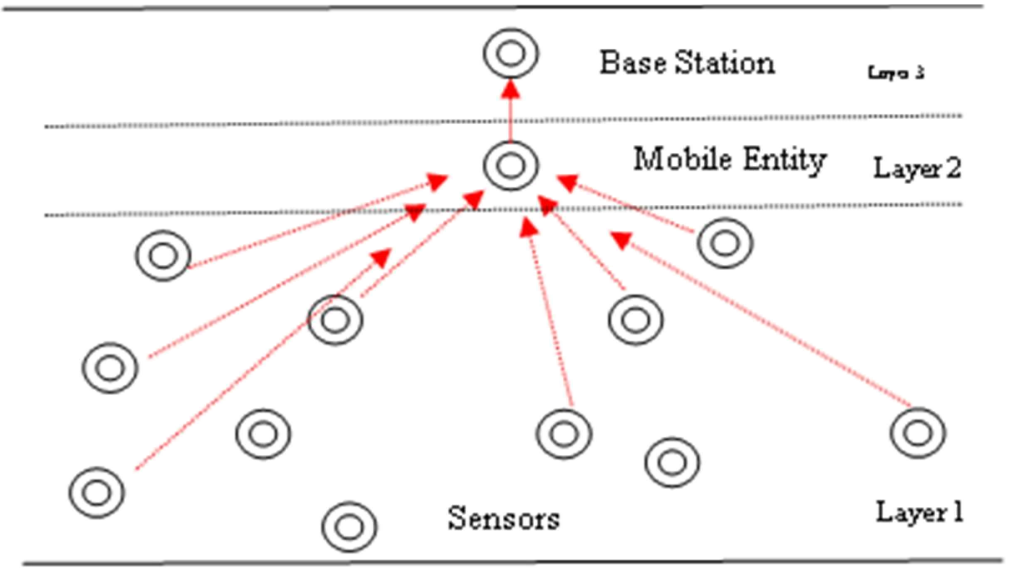

Fig. 8 Mobility with direct communication

Energy consumption in ring 0 is

Total Energy Consumption $=$ Energytrans + Energysense

$=A X H^{2} X a+E X a+\mathbb{C} X a$ where $H<r$

$=a\left(A X H^{2}+E+\mathbb{C}\right)$

(9)
Energy consumption in ring 1 is

Total Energy Consumption $=$ Energytrans + Energysense

$=A X H^{2} X a+E X a+\mathbb{C} X a$

$=a\left(\nexists X H^{2}+\mathscr{E}+\mathbb{C}\right)$ (10) 


\section{COMPARISON OF ENERGY CONSUMPTION}

This section presents outcomes of simulation presented by other researchers using the four mechanisms discussed above.

\section{$3.1 \mathrm{LEACH}$}

LEACH [13] performed simulation studies using three mechanisms, clustering mechanism, direct communication with base station and minimum energy multihop routing, and compared their performances. Based on the simulation results (Figure 9), clustering concept was found to consume lesser energy as compared to direct transmission and multihop routing. Their results also indicate that direct transmission incur higher energy as compared to multihop routing. As shown in Eq (6), energy consumption in ring 1 is $4 \mathrm{~d}^{2}$ as compared to Eq (2) where energy consumption in ring 1 is $\mathrm{d}^{2}$ only. This is because in direct transmission, each sensor node sends its data directly to the base station. If the base station is placed farther from the sensor nodes, this communication require high transmission power. In MTE (multihop), nodes route data destined for the base station through intermediate nodes (as referred to Eq 1).

Clustering $\quad------\rightarrow$ Multihop $\quad------\rightarrow$ Direct Transmission

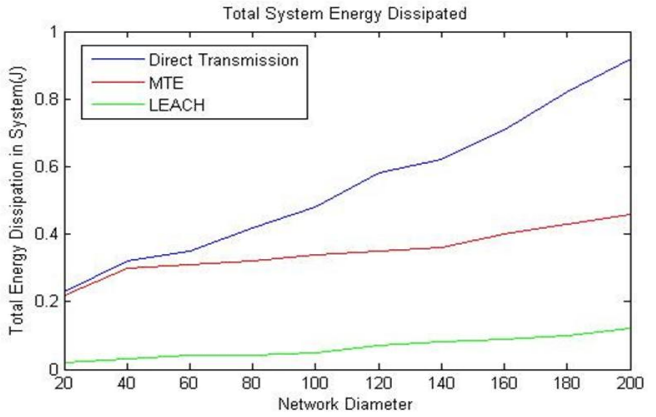

Fig. 9 System energy dissipation

\subsection{SENMA}

The following section discusses two more approaches using flatAdHoc network (multihop) and SENMA (mobile) [14]. SENMA deploys mobile agents $\mathrm{Eq}(5)$ and $\mathrm{Eq}(6)$ for communication with the base station, in which mobile agents are powerful hardware units which can be an aerial vehicle or ground vehicle. Mobile agents collect sensor data and relay to the base station, whereas
flatAdHoc architecture (Eq1) employs multihop mechanism, in which sensor

nodes are continuously consuming energy to route data through intermediate nodes. As seen in Figure 10, the deployment of mobile agent consumes lesser energy compared to flat architecture using multihop routing.

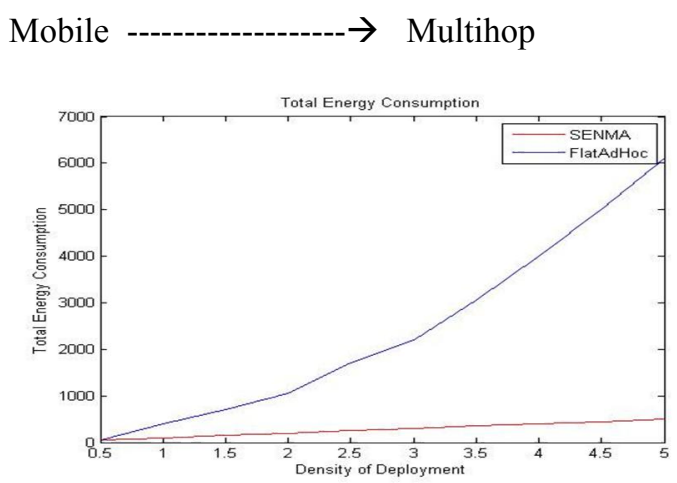

Fig. 10 Total energy consumption

\subsection{MONSOON}

MONSSON performed comparison of average power consumption for three different protocols in which LEACH is a clustering based (Eq 3) approach, MONSOON [25] is a biologically inspired framework that consists of agents and middleware platform. In the proposed framework, data collection applications is sensed by each node by deploying a mobile agent with a randomlygenerated behaviour policy. Based on the simulation results presented in Figure 11, mobility based transmission consumes lesser energy as compared to clustering.

Mobile(middleware) $------\rightarrow$ Middleware $------\rightarrow$
Clustering

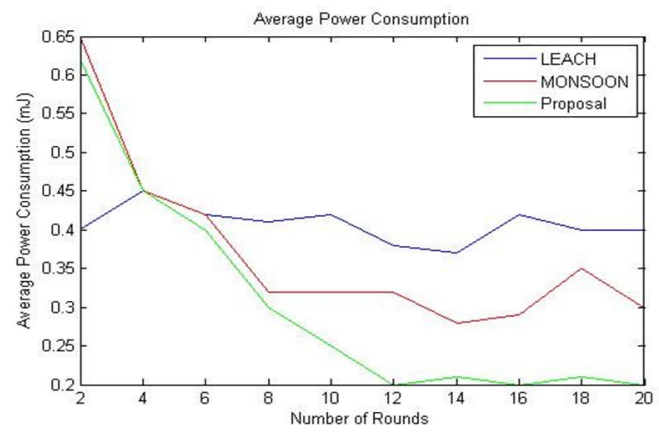

Fig. 11 Average Power Consumption

Based on our mathematical analysis and the three different results presented by the previous researchers, the order of energy consumption rate in increasing order: 
A. Magdy et. al / International Journal of Computer Networks and Communications Security, 2 (9), September 2014
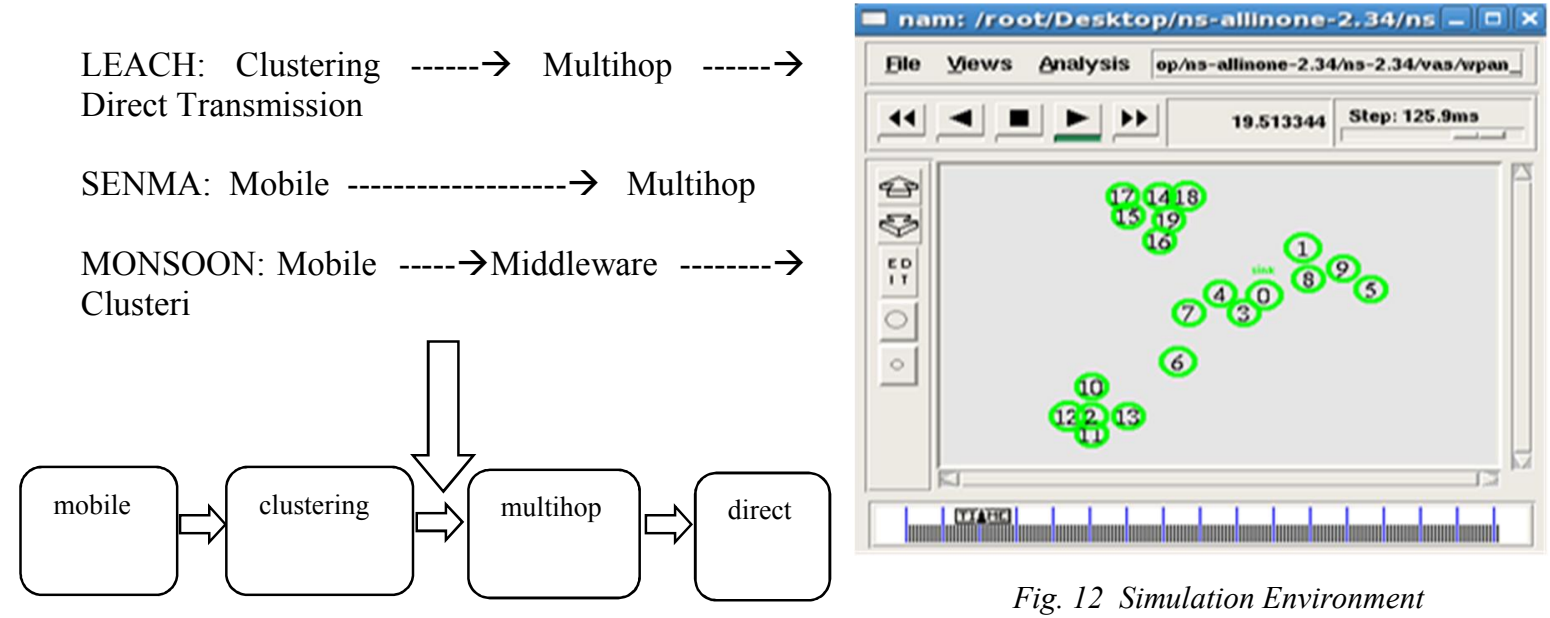

Fig. 12 Simulation Environment

\subsection{Simulation Results}

Simulation studies of the four mechanisms discussed above were conducted using NS-2 in order to evaluate the performance of our mathematical analysis (Figure 12). A simplified energy model without considering the MAC or physical layer was used in the simulation and 20 sensor nodes were scattered across an area of $670 \times 670 \mathrm{~m} 2$. The simulation was conducted by first configuring the network into multihop architecture and AODV routing algorithm was performed to analyse the effect of multihop routing. A second simulation was then performed using the direct approach mechanism in which the sensor nodes directly forward data to the base station. Clustering was employed by dividing the nodes into different clusters and electing one node as a cluster head. Finally, mobility mechanism was used in the simulation by employing one single node as the mobile agent that moves around the simulation environment collecting data from sensor nodes. The simulation results are presented in Figure 13 and Figure 14. The simulations produce similar results as our mathematical analysis and also the simulated results by LEACH, SENMA and MONSOON. Therefore, we can conclude that mobility consumes the least energy followed by clustering, multihop and finally direct transmission.

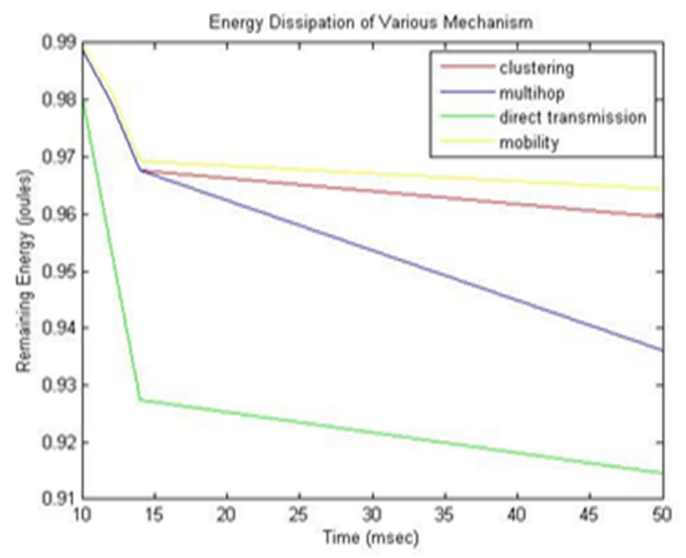

Fig.13 Energy dissipation for various mechanisms

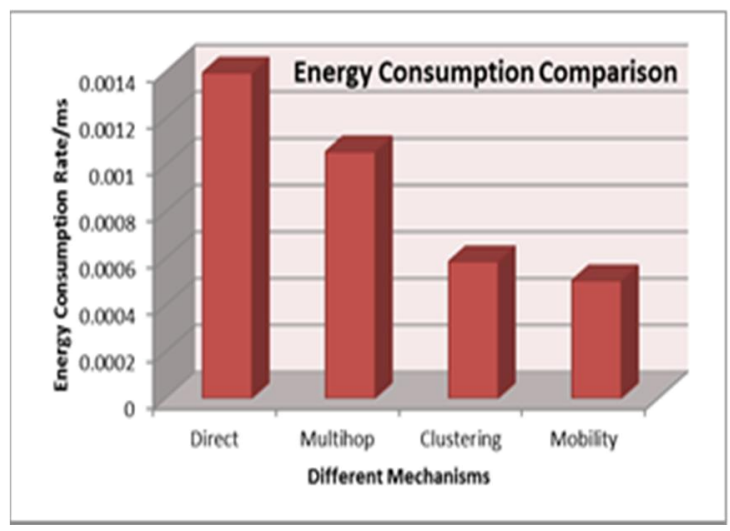

Fig. 14 Energy consumption comparison 


\section{CONCLUSION}

The findings show that direct transmission incurs the highest energy followed by multihop communication and clustering. Mobile communication consumes the least energy compared to other mechanisms. Direct transmission is feasible when the base station and sensor nodes are within close proximity as total energy consumption is proportional to the distance. In a wider network, direct transmission will not be a suitable choice as nodes will be consuming more energy and will eventually die. For wider networks, multihop can be a viable solution as this communication ensures delivery of data to the base station. But one potential problem with multihop communication concerns nodes that are closer to the base station; these nodes tend to be heavily utilized and will eventually cause routing hole near the base station. Our mathematical analysis in $\mathrm{Eq}(1)$ and $\mathrm{Eq}(9)$ reveals that energy consumption using multihop is two times more than mobile communications. This is further validated by $[23$, 24] through their simulation results.

Clustering is another energy efficient protocol proposed by researchers [11], where a cluster head is used to relay data to the base station. Clustering performs better than multihop and direct communication in terms of energy utilization. This is because cluster head selection allows other sensor nodes to only sense and relay data to the base station rather than routing data from other nodes (as in multihop). Clustering works efficiently with the rotation of cluster head election and in a smaller network. However, the performance of efficient energy utilization in clustering drops with the increase in network size as nodes have to communicate with the cluster head from time to time. Since cluster head communicates directly with the base station, more energy is utilized for the communication as discussed earlier. In order to overcome the weaknesses discussed above, researchers are proposing mobility based communication in which mobile entities will be moving closer to the base station to relay data. This reduces the transmission distance between the sender and receiver.

\section{REFERENCES}

[1] S. Sudevalayam and P. Kulkarni. (2008). Energy Harvesting Sensor Nodes: Survey and Implications.
[2] [2] N. Al-Karaki, A.E. Kamal. (2004). Routing techniques in wireless sensor networks: a survey, IEEE Wireless Commun. 11 6-28.

[3]

[4] [3] K. Sha, J. Du, and W. Shi. (2005). Wear: A balanced, fault-tolerant, energy- aware routing protocol for wireless sensor networks, Technical Report MIST-TR-2005-001, Wayne State University.

[5] [4]Yu, D. Estrin, and R. Govindan. (2001). Geographical and Energy-Aware Routing: A Recursive Data Dissemination Protocol for Wireless Sensor Networks, UCLA Computer Science Department. Technical Report, UCLACSD TR-01-0023.

[6] [5] K. Akkaya and M. Younis. (2004). Energy-Aware Routing to a Mobile Gateway in Wireless Sensor Networks, In Proceedings of the IEEE Globecom Wireless Ad Hoc and Sensor Networks Workshop, Dallas, TX.

[7] [6] JS Liu and CH Lin. (2003). Power efficiency clustering method with power limit constraint for sensor networks performance, Proceedings of the 2003 IEEE international performance, computing, and communications conference, vol. 9, Arizona, USA, p. 129-36.

[8] [7] B. Priscilla and E. Callaway. (2002). Energy efficient system design with optimum transmission range for wireless ad-hoc networks, Proceedings of the 2002 IEEE international conference on communications, vol. 2, New York, USA,. p. 945-52.

[9] [8] W. Heinzelman, A. Chandrakasan, and H. Balakrishnan. (2002). AnApplicationspecific Protocol Architecture for Wireless Microsensor Networks, IEEE Transactions on Wireless Communications 2002;1(4):660-70.

[10] [9] J. Li and P. Mohapatra. (2007). Analytical modeling and mitigation techniques for the energy hole problems in sensor networks, Pervasive and Mobile Computing 3(8) (2007) 233-254.

[11] [10] B.Przdatek, D.Song and A.Perrig. (2003). Sia:Secure information aggregation in sensor networks, Proceedings of the ACM SenSys 2003, Los Angeles, CA, November 2003.

[12][11] K. Asfandyar Khan, A. Azween and A. Rahman. (2009). Data Delivery Optimization By Efficient Cluster Head Selection of Wireless Sensor Network, 5th International Colloquium on Signal Processing\&Its Applications(CSPA), 2009, pp.186-190.

[13][12] T.J Low and A. Abdullah. (2011). Underwater Acoustic Communications: 
Optimizing Data Packet Size With Respect to Throughput Efficiency, BER, and Energy Efficiency. 2011 International Conference on Telecommunication Technology and Applications (ICTTA 2011), 2-4 May 2011, Sydney.Proc .of CSIT vol.5 (2011) C) (2011) IACSIT Press, Singapore. pp 7-14

[14]

[15] [13] Al-Fares, Z. Sun, H. and Cruickshank. (2009). A Hierarchical Routing Protocol for Survivability in Wireless Sensor Network (WSN), Proceedings of the International MultiConference of Engineers and Computer Scientists, IMECS 2009, Vol 1.

[16][14] L. Tong, Q. Zhao, and S. Adireddy. (2007). Sensor Networks with Mobile Agents, in Proc. 2003 Military Communications Intl Symp., (Boston, MA), Oct 2007.

[17][15] S. Jain, R. C. Shah, G. Borriello, W. Brunette, and S. Roy. (2004). Exploiting mobility for energy efficient data collection insensor networks, in WiOpt.

[18] [1 [16] P. Juang, H. Oki, Y. Wang, M. Martonosi, L. S. Peh, and D. Rubenstein. (2002). Energy efficient computing for wildlife tracking: design tradeoffs and early experiences with zebranet, In ASPLOS-X: Paper presented at the 10th international conference on Architectural support for programming languages and operating systems, pp. 96-107.

[19] [17] H. Jun, W. Zhao, M. Ammar, E. Zegura and C. Lee. (2005). Trading Latency for Energy in Wireless Ad Hoc Networks using Message Ferrying, Proceedings of the IEEE PerCom Workshops, International Workshop on Pervasive Wireless Networking (PWN 2005).

[20] [1 [18] R. Shah, S. Roy, S. Jain, W. Brunette. (2003). Data MULEs: Modeling a Three-tier Architecture for Sparse Sensor Networks, IEEE SNPAWorkshop.

[21] [19] M. Shakya, J. Zhang, P. Zhang, and M. Lampe. Design and optimization of wireless sensor network with mobile gateway. In International Conference on Advanced Information Networking and Applications Workshops, 2007.

[22][20] C. Perkins, E .Belding-Royer, S. Das. RFC 3561-ad hoc on-demand distance vector (AODV) routing [Online], available at : http://www.faqs.org/rfcs/rfc3561.html.

[23][21] D. Ganesan, R. Govindan, S. Shenker, and D. Estrin. (2001). Highly-resilient, energy-efficient multipath routing in wireless sensor networks, Proceedings of the ACM SIGMOBILE Mobile Computing and Communications Review, vol. 5, no. 4, pp. 1125.

[24] [22] C. Intanagonwiwat, R. Govindan, and D. Estrin. (2000). Directed diffusion: a scalable and robust communication paradigm for sensor networks, Proceedings of the ACM MobiCom '00, Boston, MA, 2000, pp. 56-67.

[25] [23] V. Ponnusamy, A. Abdullah, and G.D. Alan. (2010). A Biologically based Wireless Sensor Network, Proceedings of the Global Conference on Power Control and Optimization, Kuching, December.

[26][24] V. Ponnusamy and A. Abdullah. (2011). Energy Efficient Mobility in Wireless Sensor Network, International Journal Multimedia and Image Processing, vol .1, pp 53-62.

[27]

[28][25] P. Boonma, and S. Junichi. (2008). MONSOON: A Coevolutionary Multiobjective Adaptation Framework for Dynamic Wireless Sensor Networks, Proceedings of the 41st Hawaii International Conference on System Sciences. 